\title{
15-Lipoxygenase-1 Is Involved in the Effects of Atorvastatin on Endothelial Dysfunction
}

\author{
Peng Zhang, Xin Xing, Chunxiao Hu, Hui Yu, Qian Dong, Guanglei Chang, \\ Shu Qin, Jian Liu, and Dongying Zhang \\ Department of Cardiology, The First Affiliated Hospital of Chongqing Medical University, No. 1 Yixueyuan Road, \\ Yuzhong District, Chongqing 400016, China
}

Correspondence should be addressed to Dongying Zhang; zdy202284@hospital-cqmu.com

Received 5 March 2016; Revised 16 May 2016; Accepted 27 June 2016

Academic Editor: Paola Tomietto

Copyright (C) 2016 Peng Zhang et al. This is an open access article distributed under the Creative Commons Attribution License, which permits unrestricted use, distribution, and reproduction in any medium, provided the original work is properly cited.

\begin{abstract}
Statins exert pleiotropic effects on endothelial cells in addition to lowering cholesterol. 15-Lipoxygenase-1 (ALOX15) has been implicated in vascular inflammation and disease. The relationship between atorvastatin and ALOX15 was investigated using a rat carotid artery balloon-injury model. Hematoxylin and eosin (HE) staining showed that ALOX15 overexpression increased the thickness of the intima-media (IMT). Immunohistochemistry and western blotting showed that atorvastatin increased the expression of cellular adhesion molecules (CAMs) but decreased the expression of endothelial nitric oxide synthase (eNOS); these effects of atorvastatin were blocked by ALOX15 overexpression. In human umbilical venous endothelial cells (HUVECs), silencing of ALOX15 enhanced the effects of atorvastatin on endothelial function. Expression levels of CAMs and Akt/eNOS/NO under oxidized low-density lipoprotein (ox-LDL) stimulation were modulated by ALOX15 inhibitor and ALOX15 small interfering RNA (siRNA). Atorvastatin abolished the activation of nuclear factor-kappa B (NF- $\kappa$ B) induced by ox-LDL. Exposure to ox-LDL induced upregulation of ALOX15 in HUVECs, but this effect was partially abolished by atorvastatin or the NF- $\kappa$ B inhibitor pyrrolidine dithiocarbamate (PDTC). These results demonstrate that regulation of ALOX15 expression might be involved in the effects of atorvastatin on endothelial dysfunction.
\end{abstract}

\section{Introduction}

Statins are widely used to treat hyperlipidemia and prevent coronary heart disease. In addition to their lipidlowering effects, statins produce an antiatherogenic effect by improving endothelial function, stabilizing atherosclerotic plaques, and reducing oxidative stress and endothelial inflammation [1-3]. In addition, statins enhance expression of growth factors, activate the phosphatidylinositol 3-kinase (PI3K)/Akt-mediated signaling pathway, and postpone the initiation of atherosclerosis through decreased regulation of nitric oxide (NO) synthesis by upregulating endothelial nitric oxide synthase (eNOS) mRNA expression and decreasing superoxide anion $\mathrm{O}_{2}$-production in endothelial cells $[4,5]$. Pretreatment of endothelial cells with atorvastatin reduced expression of intracellular adhesion molecule-1 (ICAM-1), cluster of differentiation 36 (CD36), von Willebrand factor (vWF), vascular cell adhesion molecule-1 (VCAM-1), cluster of differentiation 31 (CD31), and E/P-selectin [6,7]. Therefore, activation of the PI3K/Akt/eNOS pathway and downregulation of adhesion molecule expression might be involved in the beneficial effects of statins, but the mechanism by which statins improve endothelial function has not been fully elucidated.

Lipoxygenases (LOXs) constitute a heterogeneous family of lipid-peroxidizing enzymes that catalyze the oxidation of polyunsaturated fatty acids [8]. In mammals, LOXs are named according to the genes of the human ortholog. The human genome involves six functional LOX genes (ALOX15, ALOX15B, ALOX12, ALOX12B, ALOXE3, and ALOX5) [9, 10]. There is compelling evidence for a proinflammatory effect of ALOX15 through the formation of ox-LDL which accelerates foam cell formation and through its role in signaling of angiotensin II mediated mechanisms and vascular smooth muscle cell proliferation [11, 12]. ALOX15 and linoleic acid metabolite 13-S-hydroxyoctadecadienoic acid 
(13-HODE) mediate monocyte adhesion onto the blood vessel wall by activating protein kinase $\mathrm{C}$ (PKC) and expression of cellular adhesion molecules (CAMs) [13, 14]. Moreover, $13-\mathrm{HODE}$ exerts a proinflammatory effect via transcription factors such as nuclear factor-kappa B (NF- $\kappa \mathrm{B})$ [15]. ALOX15 activation is involved in the pathogenesis of atherosclerosis, hypertension, and diabetes [16]. Deletion of ALOX15 alters macrophage and islet function in nonobese diabetic ALOX15 (null) mice, leading to decreased mRNA and protein levels of proinflammatory cytokines and preventing the development of type 1 diabetes [17]. Inhibition of the initial phase of the development of atherosclerosis by statins may reduce lipid peroxidation during atherosclerotic plaque formation [18]. ALOX15 might participate in the mechanism by which statins improve endothelial function because ALOX15 is a key enzyme involved in lipid oxidation; however, little is known about the effect of atorvastatin on ALOX15.

In this paper, we identify ALOX15 as a new mediator of the effects of statins on endothelial function.

\section{Materials and Methods}

Detailed materials and methods are described in the Supplementary Material available online at http://dx.doi.org/10.1155/ 2016/6769032.

2.1. Construction of siRNA and Lentivirus Encoding ALOX15. ALOX15 shRNA lentiviral particles were purchased from Santa Cruz Biotechnology, Inc. (Santa Cruz, CA, USA). Human ALOX15 cDNA (accession number NM-001140) was subcloned into the NotI/NsiI site of pLV5-GFP (GenePharma, Shanghai, China). Rat ALOX15 cDNA (accession number NM-031010) was subcloned into the AgeI/AgeI site of pGV287-EGFP (GeneChem, Shanghai, China). The presence of both genes was confirmed by sequencing. The resulting recombinant vector was transfected into $293 \mathrm{~T}$ cells with Lipofectamine ${ }^{\circledR} 2000$ Plus (Invitrogen, CA, USA) in order to package viral particles expressing ALOX15. Green fluorescent protein- (GFP-) expressing lentivirus was used as a control.

2.2. Animal Models of Carotid Balloon Injury and Lentiviral Gene Transfer. Ten-week-old male Sprague-Dawley rats weighing 380-420 g (Animal Experiment Center of Chong Qing Medical University) were utilized. All animals were housed under conventional conditions in the animal care facility. All animals received humane care in compliance with the Principles of Laboratory Animal Care formulated by the National Society for Medical Research and the Guide for the Care and Use of Laboratory Animals. The animal experimental protocol was approved by the Animal Care Committee of The First Affiliated Hospital of Chongqing Medical University. Rats were fed standard laboratory chow and tap water ad libitum. Experiments were performed under general anesthesia achieved by an injection of ketamine $(80 \mathrm{mg} / \mathrm{kg})$ and xylazine $(5 \mathrm{mg} / \mathrm{kg})$. The rat carotid injury model was performed as described previously [19]. The left common carotid artery was denuded of endothelium by the intraluminal passage of a $2 \mathrm{~F}$ balloon catheter (Fogarty, Edwards Lifesciences, LLC, Irvine, CA, USA) introduced through the external carotid artery. During denudation of the endothelium, the balloon was inflated with $0.02 \mathrm{~mL}$ of $0.9 \%$ sodium chloride (saline) and then withdrawn through the common carotid artery to the carotid bifurcation with constant rotation. The denudation procedure was repeated two additional times to ensure complete endothelial denudation. Next, heparin (200 units/kg) was intraperitoneally injected to prevent thrombus formation. The protocol used for introducing lentiviruses into balloon-injured carotid arteries has been previously described [20]. The isolated distal segment of the injured artery from the proximal edge of omohyoid muscle to the carotid bifurcation was washed with $200 \mu \mathrm{L}$ of saline and incubated with lentivirus $\left(1.0 \times 10^{8} \mathrm{TU} / \mathrm{mL}\right)$ expressing either GFP or ALOX15 for 20 minutes. Seven days or fourteen days later, the balloon-injured, lentivirus-inoculated segment was perfused with saline and removed. For the atorvastatin group, atorvastatin ( $4 \mathrm{mg} / \mathrm{kg}$ per day) was orally administered daily to the rats for 14 days beginning shortly after the operation.

2.3. Morphometric Analysis of Immunohistochemistry. To assess the inflammatory process and endothelial integrity, protein expression levels of VCAM-1, ICAM-1, and eNOS were assayed by western blot analysis.

2.4. Cell Culture. HUVECs were purchased from Nanjing KeyGen Biotech and cultured in Roswell Park Memorial Institute- (RPMI-) 1640 medium with 10\% fetal bovine serum at $37^{\circ} \mathrm{C}$ in an incubator containing $5 \% \mathrm{CO}_{2}$. The fourth to eighth passages of HUVECs were used in all experiments.

2.5. Enzyme Immunoassay Measurement. Frozen tissue samples were homogenized for one minute on low speed in cold PBS. 13(S)-HODE from tissue samples and HUVECs were extracted by ice cold lysis buffer. An aliquot was reserved for protein determination (BCA). The organic phase of the solution was extracted using 3-fold excess of saturated ethyl acetate. Pooled organic phase solutions were dried completely under cold temperature. Residues were dissolved in $25 \mu \mathrm{L}$ of ethanol and $125 \mu \mathrm{L}$ of assay buffer (provided with EIA kits) for EIAs. 13(S)-HODE levels were measured in tissues and cells by use of a commercially available enzyme immunoassay (EIA) kit (Novus Biologicals).

2.6. Quantitative Real-Time Reverse-Transfection PCR. Total cellular RNA was isolated from tissue samples and HUVECs using TRIzol (Invitrogen, USA) and the reverse transcription reaction was performed with $1 \mu \mathrm{g}$ RNA in $20 \mu \mathrm{L}$ reaction using PrimeScript ${ }^{\mathrm{TM}} \mathrm{RT}$ reagent kit (Takara, Japan) at $42^{\circ} \mathrm{C}$ for $2 \mathrm{~min}$ and $37^{\circ} \mathrm{C}$ for $15 \mathrm{~min}$ followed by $85^{\circ} \mathrm{C}$ for $5 \mathrm{sec}$. The $\mathrm{qPCR}$ was performed on a StepOnePlus ${ }^{\mathrm{TM}}$ Real-Time PCR System (Applied BioSystems, USA) using SYBR Green method with the following parameters: preheating for $30 \mathrm{sec}$ at $95^{\circ} \mathrm{C}$, followed by 45 cycles of denaturation for $5 \mathrm{sec}$ at $95^{\circ} \mathrm{C}$, and annealing for $34 \mathrm{sec}$ at $60^{\circ} \mathrm{C}$. Primers were designed using Primer-Blast (http://www.ncbi.nlm.nih.gov/tools/primerblast/) and synthesized by Sangon Biotech (Shanghai, China). All mRNA expression levels were normalized to those of 
GAPDH. The primer sequences used were rat ALOX15 (forward: GTCTACTCCACCACCTATTTTC, reverse: CTGTGCTCATTGCCTTGTC) and human ALOX15 (forward: GGAGCCTTCCTAACCTACAGC, reverse: CTCACGATTCCTTCCACATACC).

2.7. Western Blotting Analysis. Protein expression levels of ALOX15, VCAM-1, ICAM-1, nuclear factor of kappa light polypeptide gene enhancer in B-cells inhibitor alpha (I $\kappa \mathrm{B}$ $\alpha$ ), NF- $\kappa$ B-p65, Akt, p-Akt (Ser473), eNOS, and p-eNOS (Ser1177) were assayed by western blot analysis.

2.8. Statistical Analysis. All values are expressed as mean \pm SEM. Comparisons between groups were performed using one-way analysis of variance (ANOVA) followed by the Student-Newman-Keuls (SNK) test or Dunnett's test using SPSS 17.0 software (IBM Corp., Armonk, NY, USA). $P$ values less than 0.05 were considered statistically significant.

\section{Results}

3.1. Gene Transfer Efficacy and Effect of ALOX15 on Endothelial Proliferation In Vivo. To assess the efficacy of in vivo gene transfer using GFP-conjugated lentiviral constructs, the distal segments of injured left carotid arteries were incubated with saline, Lv-GFP, or Lv-ALOX15 for 20 min immediately after the balloon injury was performed. After 7 or 14 days, the artery segments were harvested, fixed, paraffin-embedded, and sectioned. Because GFP expression provides a convenient way to test the transfection efficiency of lentivirus [21], GFP fluorescence in the carotid endothelium was examined. GFP fluorescence observations suggested successful transfection of ALOX15 at 14 days (Figure 1(a)). qRT-PCR showed no significant difference between the ALOX15 mRNA expression levels of the Lv-GFP-transduced and Lv-ALOX15transduced vessels (Figures S1(a) and 1(b) in the online-only Supplementary Material). Western blotting showed no significant difference between the ALOX15 expression levels of the saline-treated and Lv-GFP-transduced vessels. However, ALOX15 expression was markedly increased in Lv-ALOX15transduced vessels in comparison with that of the saline or Lv-GFP-incubated vessels (Figure 1(b), Table 1, $P<0.001$ ). Enzyme immunoassays showed that 13-HODE expression was markedly increased in Lv-ALOX15-transduced vessels in comparison with that of the saline or Lv-GFP-incubated vessels (Figure $1(\mathrm{c})$, Table 1, $P<0.01$ ). HE staining was used to test the effect of ALOX15 on endothelial proliferation. The distal segments of the injured left carotid arteries were harvested, fixed, paraffin-embedded, and sectioned 14 days after the balloon injury was performed. As shown in Figures 1(d) and 1(e) and Table 1, balloon injury significantly increased IMT in comparison with that of the Sham group $(P<$ 0.001). Interestingly, Lv-ALOX15 significantly increased IMT in comparison with Lv-GFP $(P<0.005)$. The Lv-GFP- and saline-treated groups did not show a significant difference in IMT following balloon injury. These results indicate that the lentiviral construct system is an effective in vivo delivery method for inducing ALOX15 protein expression and activity in the carotid artery. Importantly, ALOX15 overexpression
TABLE 1: Numbers in results.

\begin{tabular}{lcc}
\hline Figure 1 & Group & Mean \pm SD \\
\hline (b) & Lv-ALOX15 & $2.479 \pm 0.526$ \\
\hline (c) & Lv-ALOX15 & $31.528 \pm 9.632$ \\
\hline (e) & BI & $0.132 \pm 0.012$ \\
& BI + Lv-ALOX15 & $0.147 \pm 0.010$ \\
\hline
\end{tabular}

TABLE 2: Numbers in results.

\begin{tabular}{lcc}
\hline Figure 2 & Group & Mean \pm SD \\
\hline (b) & BI + Ator & $0.977 \pm 0.059$ \\
& $\mathrm{BI}+$ Ator + ALOX15 & $0.494 \pm 0.038$ \\
\hline \multirow{2}{*}{ (c) p-AKT } & BI + Ator & $0.889 \pm 0.192$ \\
& $\mathrm{BI}+$ Ator + ALOX15 & $0.344 \pm 0.054$ \\
\hline \multirow{2}{*}{ (c) p-eNOS } & BI + Ator & $0.667 \pm 0.161$ \\
& $\mathrm{BI}+$ Ator + ALOX15 & $0.319 \pm 0.092$ \\
\hline \multirow{2}{*}{ (d) VCAM-1 } & $\mathrm{BI}+$ Ator & $1.002 \pm 0.183$ \\
& $\mathrm{BI}+$ Ator + ALOX15 & $1.538 \pm 0.138$ \\
\hline \multirow{2}{*}{ (d) ICAM-1 } & BI + Ator & $1.417 \pm 0.067$ \\
& $\mathrm{BI}+$ Ator + ALOX15 & $1.770 \pm 0.128$ \\
\hline
\end{tabular}

induced by Lv-ALOX15 enhanced endothelial proliferation in the rat carotid artery balloon injury model.

3.2. ALOX15 Overexpression Reduced the Protective Role of Atorvastatin In Vivo. Interventional vascular procedures such as balloon angioplasty and stent placement have been shown to result in greater restenosis rates in the clinic [22]. These vascular procedures cause mechanical injury to the artery, leading to a cascade of inflammatory events and inactivation of vascular factors, but statins partially inhibit such responses of the endothelium to injury [23$25]$. To test the effects of atorvastatin on endothelial injury and assess the participation of ALOX15 in such effects, we examined Akt/eNOS expression, phosphorylation of Akt ( $\mathrm{p}$ $\mathrm{Akt}^{\mathrm{Ser} 473}$ ) and eNOS (p-eNOS ${ }^{\mathrm{Ser1177}}$ ), and VCAM-1/ICAM1 in balloon-injured carotid arteries using immunohistochemistry and western blotting. As shown in Figure 2(a) and Table 2, the groups subjected to vascular balloon injury showed significantly reduced levels of total eNOS, but increased levels of VCAM-1/ICAM-1, in comparison with those of the Sham group. In the injured arteries, atorvastatin treatment significantly increased downregulation of eNOS but significantly decreased upregulation of VCAM-1/ICAM1; however, the group treated with Lv-ALOX15 showed significantly decreased eNOS abundance, but significantly increased VCAM-1/ICAM-1 abundance, in comparison with the levels of these proteins in the group treated with Lv-GFP. Consistently, western blot analysis showed that atorvastatin treatment increased total eNOS protein expression $(P<$ $0.001)$, as well as expression of $\mathrm{p}-\mathrm{Akt}^{\mathrm{Ser} 473} / \mathrm{p}-\mathrm{eNOS}^{\operatorname{Ser} 1177}$ $(P<0.05)$, while it decreased protein levels of VCAM1 and ICAM-1 $(P<0.001)$. These results indicate that atorvastatin might regulate the activity of eNOS through modulating phosphorylation at these sites. Moreover, the group treated with Lv-ALOX15 showed decreased protein 

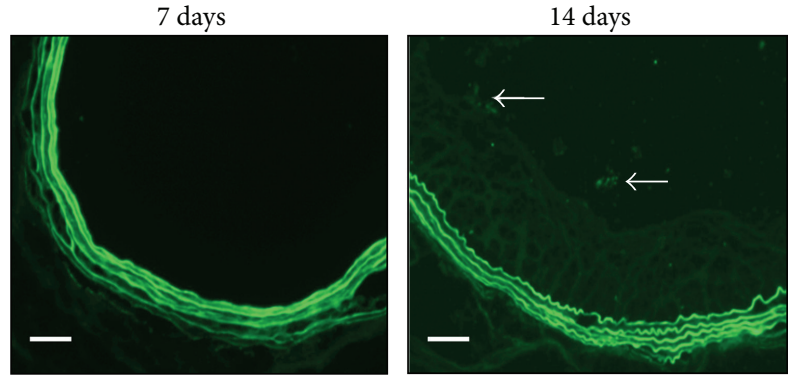

(a)
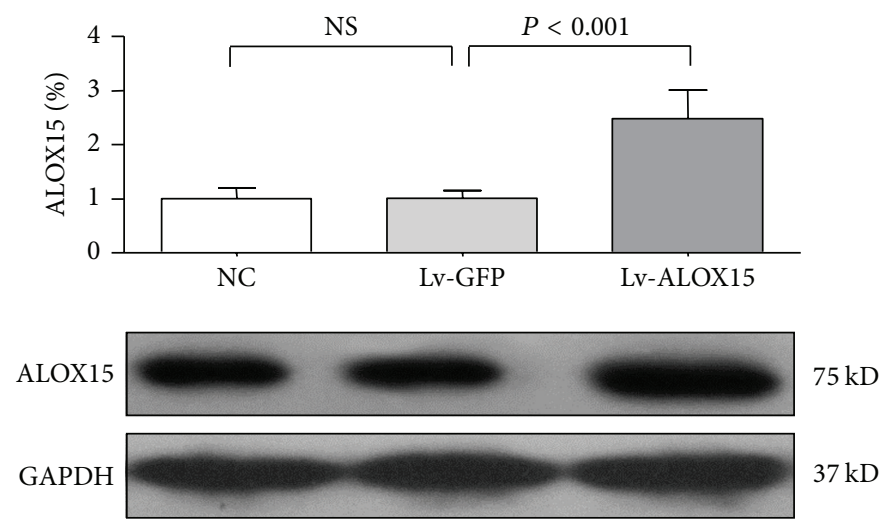

(b)

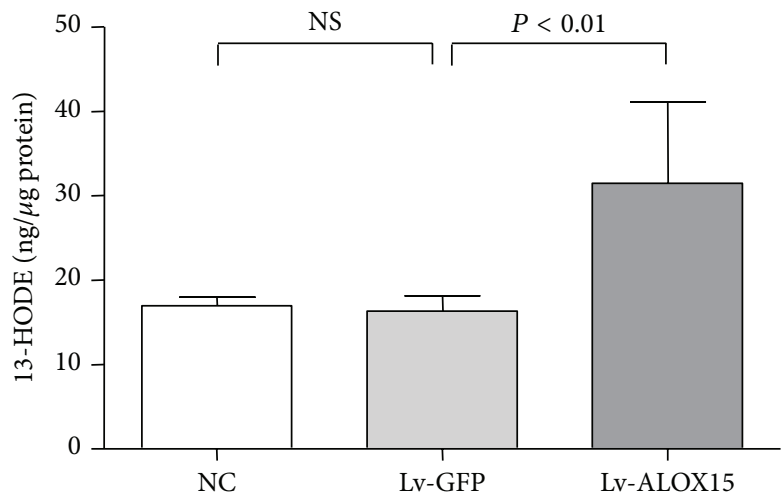

(c)
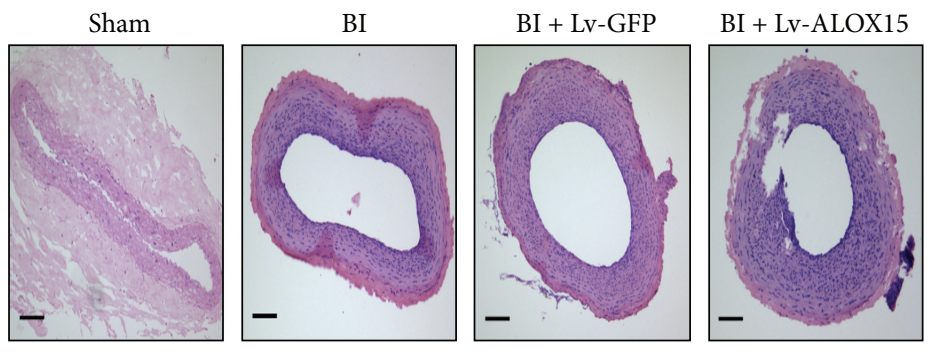

(d)

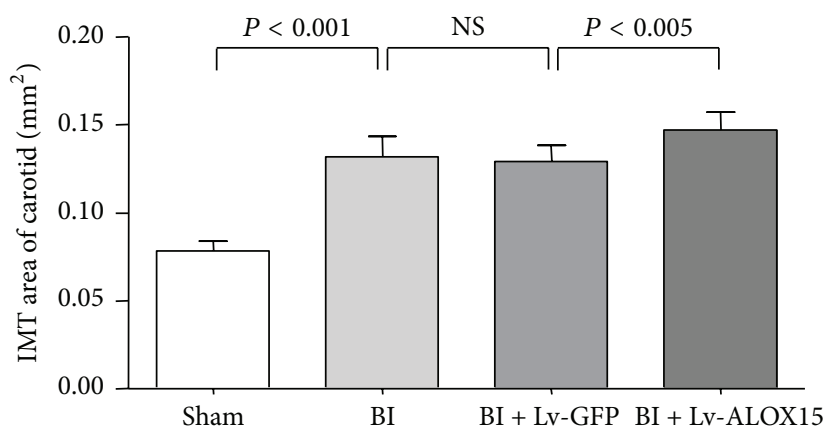

(e)

FIGURE 1: Lentivirus-based ALOX15 overexpression in the rat carotid artery and the effect of ALOX15 on neointima formation in rat ballooninjured (BI) carotid arteries. (a) Representative images showing no detectable green fluorescence after 7 days, but pronounced fluorescence after 14 days, in the neointima of carotid arteries after transfection with lentiviral constructs coexpressing GFP. (b) Western blot analyses showed that Lv-ALOX15 upregulated ALOX15 protein expression. (c) EIAs showed that Lv-ALOX15 upregulated linoleic acid metabolite 13HODE. (d) Immediately after BI, arteries were treated with normal saline, Lv-GFP, or Lv-ALOX15. Representative photomicrographs of carotid arteries 14 days after BI. The artery cross sections were stained with HE. (e) Quantitative analysis of IMT. The arrows indicate endothelial cells expressing GFP. The scale bar represents $100 \mu \mathrm{m}$. Results are expressed as mean \pm SD. NS: not significant. $N=4$ or 6 per group.

expression of eNOS $(P<0.001)$, p-Akt ${ }^{\operatorname{Ser} 473}(P<0.001)$, and $\mathrm{p}$-eNOS ${ }^{\mathrm{Ser} 1177}(P<0.005)$, but significantly increased protein expression of VCAM-1 and ICAM-1 $(P<0.01)$, in comparison with the protein levels of the group treated with Lv-GFP (Figures 2(b), 2(c), and 2(d), Table 2). These results indicate that ALOX15 might be involved in the effects of atorvastatin on endothelial function in vivo.
3.3. ALOX15 Was Involved in Effects of Atorvastatin on Endothelial Function In Vitro. Atherosclerosis (AS) is characterized by chronic oxidative stress and inflammatory changes in vascular tissue. Increased local and systemic levels of oxLDL induce endothelial cell activation, dysfunction, apoptosis, and impaired vasorelaxation, which contribute to the development and progression of atherosclerosis [26, 27]. To 


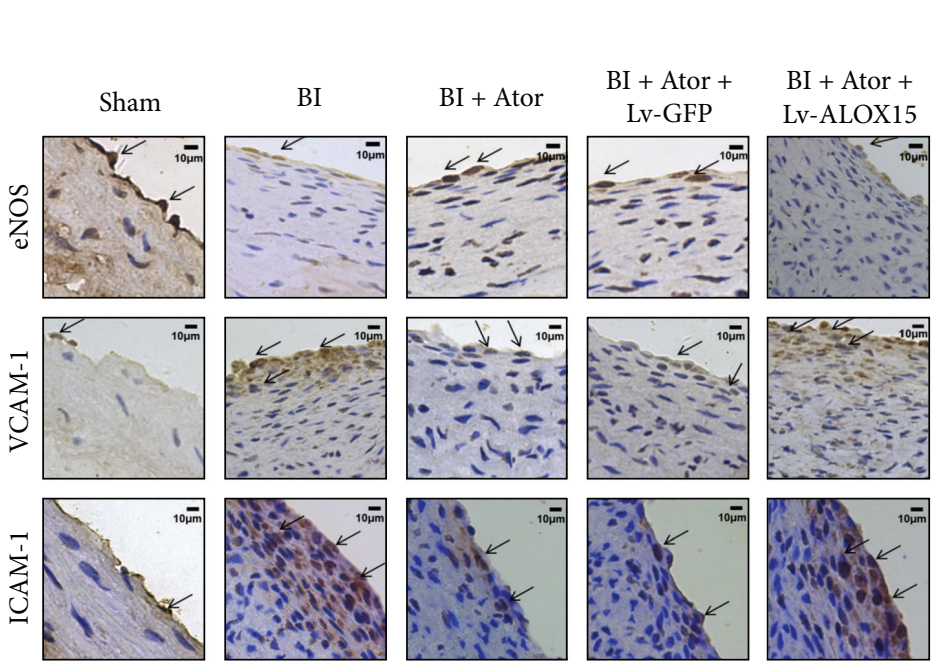

(a)
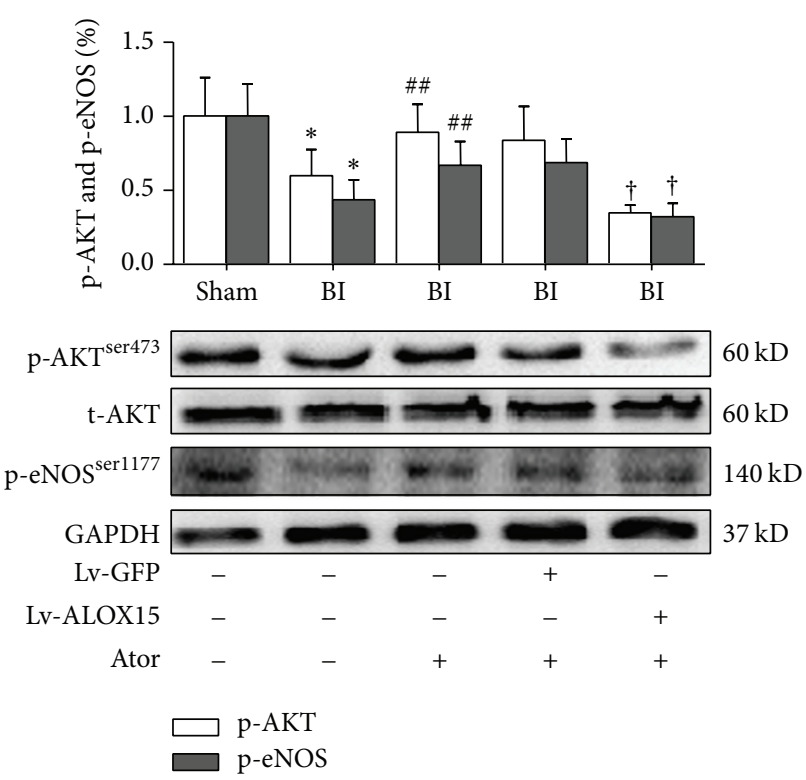

(c)
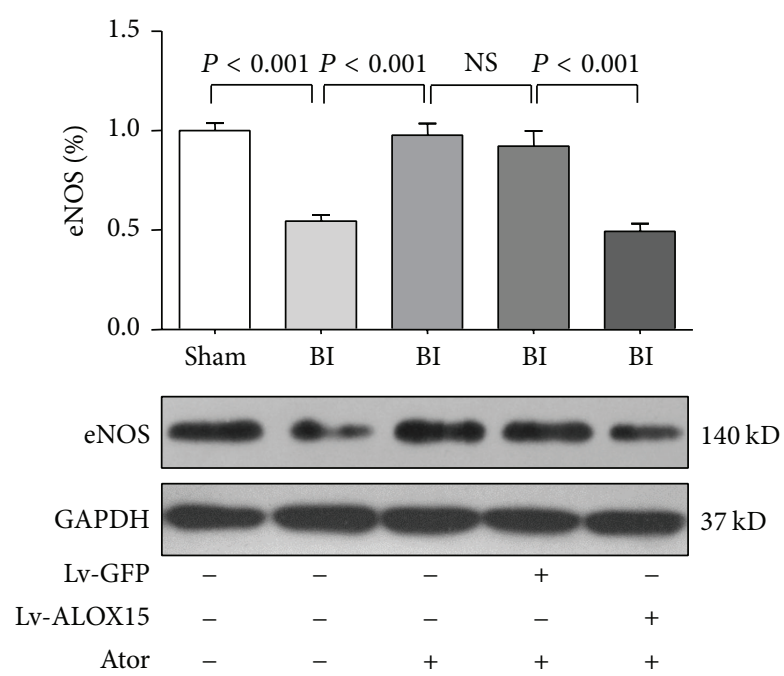

(b)
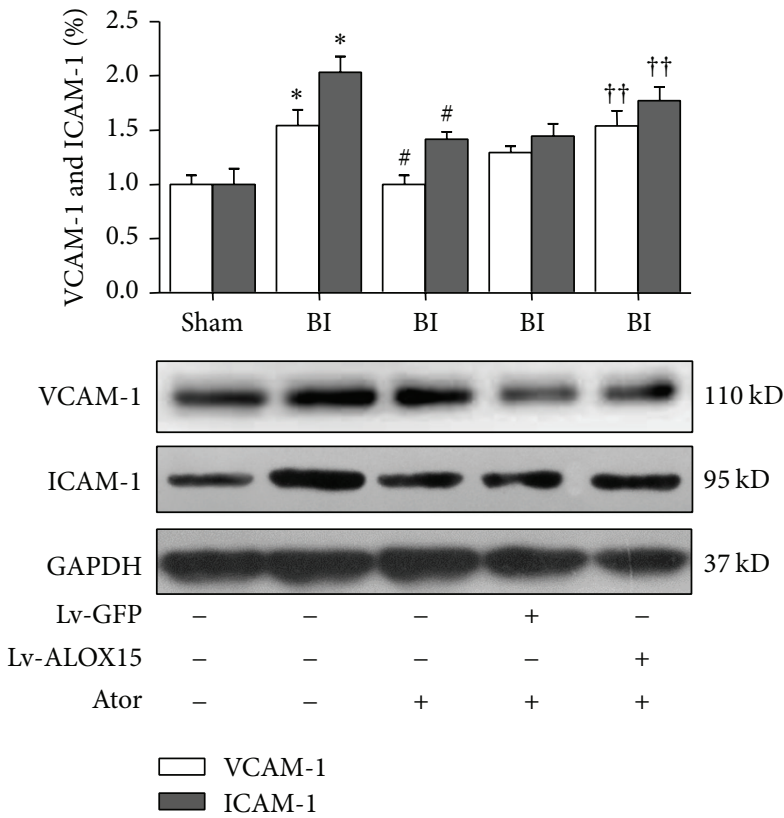

(d)

FIGURE 2: Expression levels of AKT, eNOS, VCAM-1, and ICAM-1 were modulated by lentivirus-based ALOX15 overexpression in rat ballooninjured (BI) carotid arteries 14 days after transfection. (a) Immunohistochemical analysis of eNOS, VCAM-1, and ICAM-1 in BI carotid arteries. The arrows indicate positive staining. The scale bar represents $5 \mu \mathrm{m}$. (b) Western blot analysis of eNOS expression in BI carotid arteries. (c) Western blot analysis of p-AKT/p-eNOS expression in BI carotid arteries to assess the activity levels of AKT and eNOS. The protein level of p-eNOS was normalized against that of GAPDH because total eNOS expression was changed. (d) Western blot analysis of VCAM-1/ICAM-1 expression in BI carotid arteries. The scale bar represents $10 \mu \mathrm{m}$. Results are expressed as mean \pm SD. Ator: atorvastatin; NS: not significant. ${ }^{*} P<0.01$ versus the Sham group; ${ }^{\#} P<0.001$ and ${ }^{\# \#} P<0.05$ versus the BI group; ${ }^{\dagger} P<0.01$ and ${ }^{\dagger \dagger} P<0.05$ versus the BI + Lv-GFP group. $N=4$ per group.

further assess the role of ALOX15 in the effects of atorvastatin on endothelial function in vitro, an ox-LDL-stimulated endothelial injury model was established in HUVECs. The MTT assay was performed after HUVECs were exposed to various concentrations of ox-LDL $(50,100$, and $150 \mathrm{mg} / \mathrm{L})$ for $24 \mathrm{~h}$. ox-LDL damaged HUVECs in a concentrationdependent manner. After exposure to $50 \mathrm{mg} / \mathrm{L}$ and $100 \mathrm{mg} / \mathrm{L}$ ox-LDL, cell viability was reduced to $93.8 \%(P>0.05)$ and
$75.8 \%(P<0.001)$, respectively, of that of the control group, whereas exposure to $150 \mathrm{mg} / \mathrm{L}$ ox-LDL reduced cell viability to $41.5 \%(P<0.001)$ of that of the control group (Figure S1(c) in the online-only Supplementary Material). To assess the effects of Lv-shALOX15 and Lv-ALOX15 in vitro, GFP expression was visualized by fluorescence microscopy at 24, 48 , and $72 \mathrm{~h}$ after transfection (Figure 3(a), Table 3), whereas transfection efficiency was determined by flow cytometry 

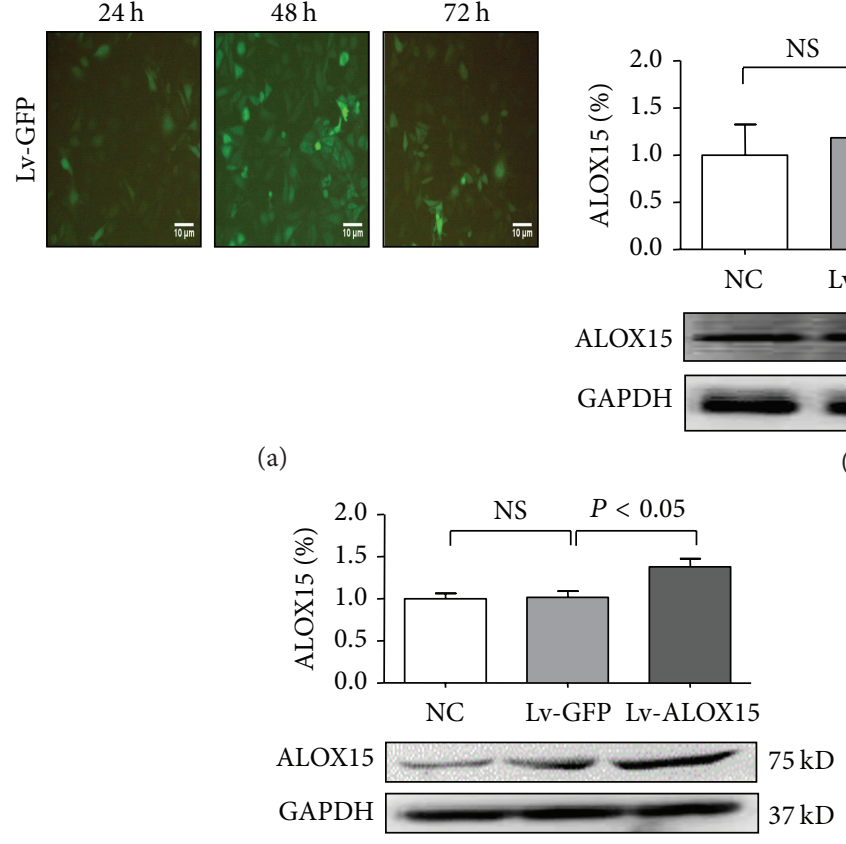

(d)

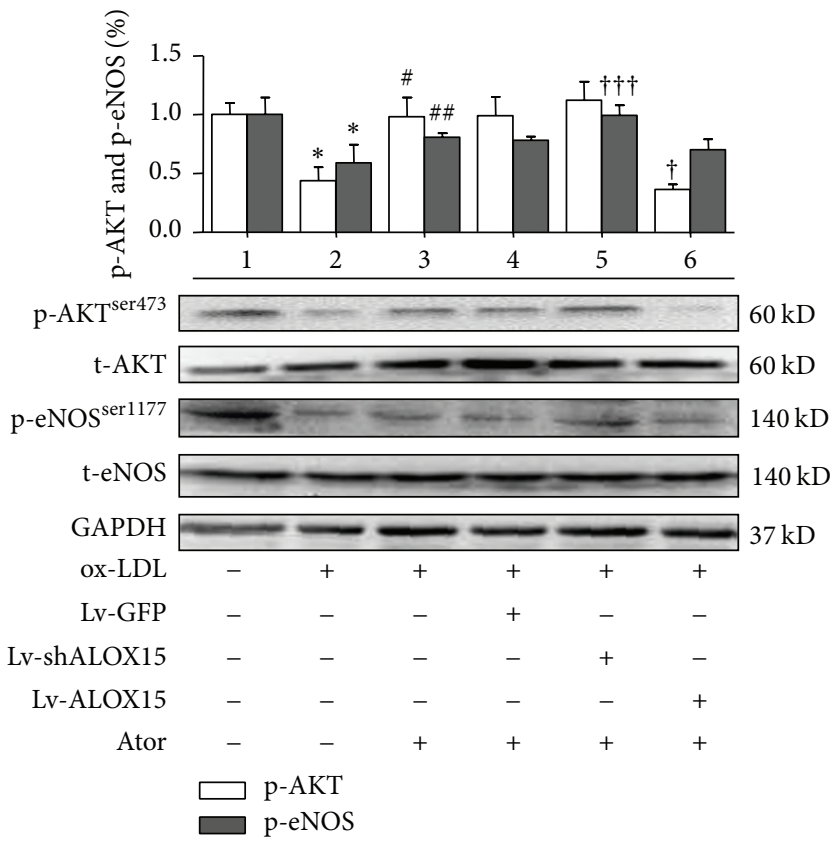

(f)
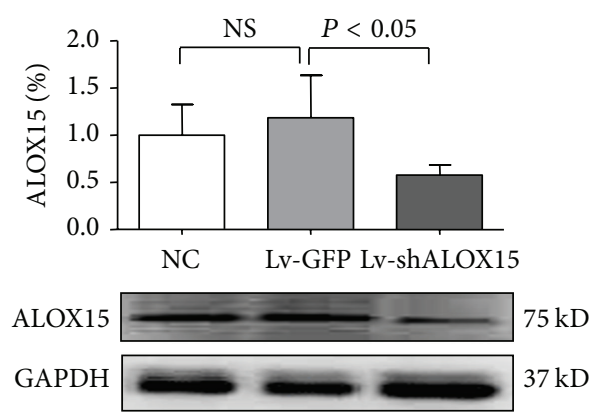

(b)
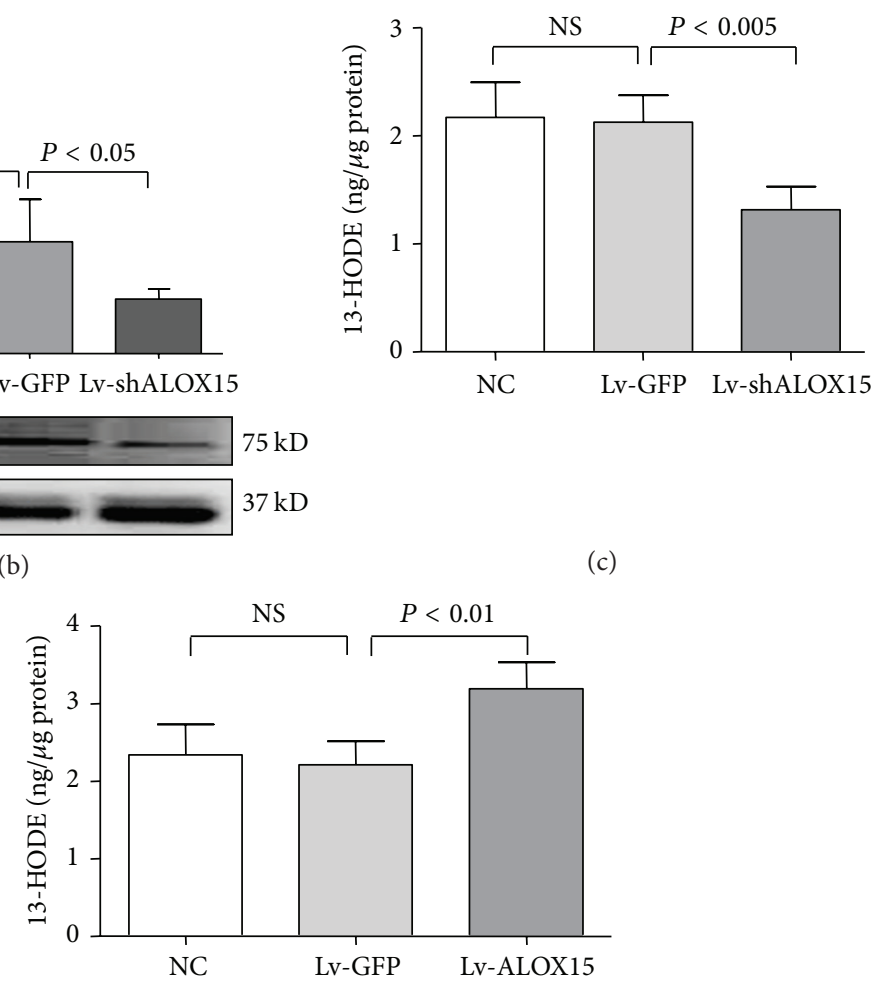

(e)

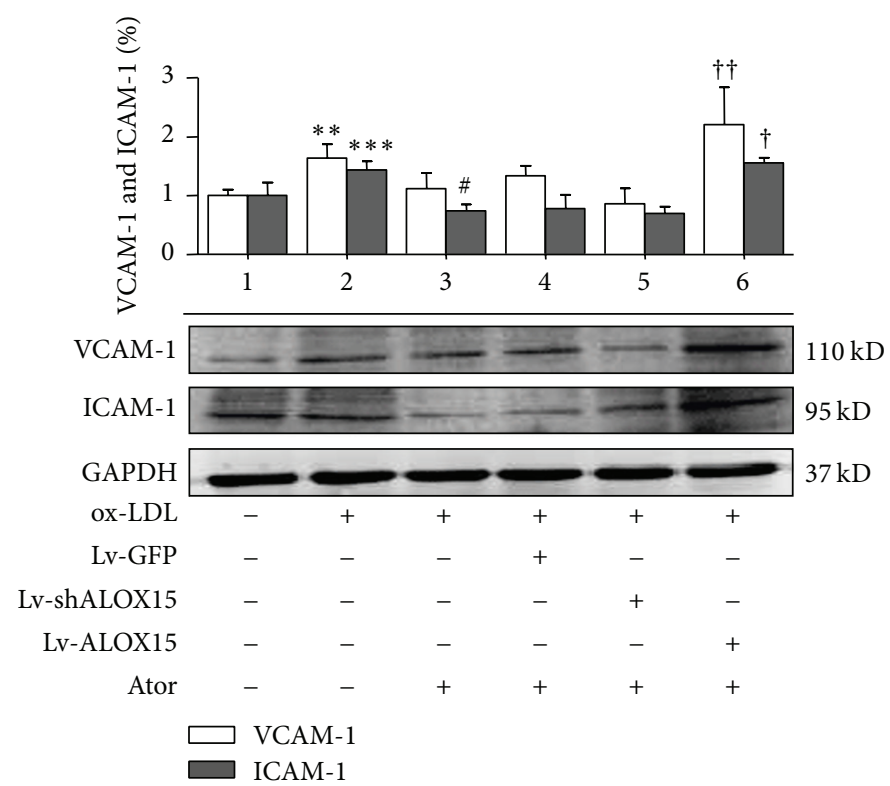

$(\mathrm{g})$

Figure 3: Changes in expression levels of ALOX15, AKT, eNOS, VCAM-1, and ICAM-1 in HUVECs $24 \mathrm{~h}$ following exposure to oxLDL $(100 \mathrm{mg} / \mathrm{L})$ or atorvastatin $(10 \mu \mathrm{M})$. (a) Photographs of Lv-GFP-transfected HUVECs. GFP expression was visualized by fluorescence microscopy $(100 \mathrm{x})$ at 24,48 , and $72 \mathrm{~h}$ after transfection. The scale bar represents $10 \mu \mathrm{m}$. (b) and (c) Western blot and EIAs indicated that Lv-shALOX15 downregulated ALOX15 protein expression and enzyme activity. (d) and (e) Western blot and EIAs indicated that Lv-ALOX15 upregulated ALOX15 protein expression and enzyme activity. (f) Western blot analysis of AKT/eNOS expression in HUVECs. (g) Western blot analysis of VCAM-1/ICAM-1 expression in HUVECs. Group 1: normal control group; Group 2: ox-LDL (100 mg/L); Group 3: oxLDL $(100 \mathrm{mg} / \mathrm{L})+$ atorvastatin $(10 \mu \mathrm{M})$; Group 4: Lv-GFP + ox-LDL $(100 \mathrm{mg} / \mathrm{L})+$ atorvastatin $(10 \mu \mathrm{M})$; Group 5: Lv-shALOX15 + ox-LDL $(100 \mathrm{mg} / \mathrm{L})+$ atorvastatin $(10 \mu \mathrm{M})$; and Group 6: Lv-shALOX15 + ox-LDL $(100 \mathrm{mg} / \mathrm{L})+$ atorvastatin $(10 \mu \mathrm{M})$. Results are expressed as mean \pm SD. NC: normal control group; NS: not significant. ${ }^{*} P<0.001,{ }^{* *} P<0.01$, and ${ }^{* * *} P<0.05$ versus Group $1 ;{ }^{*} P<0.001$ and ${ }^{\# \#} P<0.05$ versus Group 2; and ${ }^{\dagger} P<0.001,{ }^{\dagger \dagger} P<0.01$, and ${ }^{\dagger \dagger \dagger} P<0.05$ versus Group $4 . N=4$ per group. 
TABLE 3: Numbers in results.

\begin{tabular}{lcc}
\hline Figure 3 & Group & Mean \pm SD \\
\hline (b) & Lv-shALOX15 & $0.577 \pm 0.111$ \\
\hline (c) & Lv-shALOX15 & $1.315 \pm 0.215$ \\
\hline (d) & Lv-ALOX15 & $1.380 \pm 0.097$ \\
\hline (e) & Lv-ALOX15 & $3.192 \pm 0.336$ \\
\hline \multirow{3}{*}{ (f) p-AKT } & ox-LDL & $0.441 \pm 0.112$ \\
& ox-LDL + Ator & $0.981 \pm 0.163$ \\
& ox-LDL + Ator + Lv-ALOX15 & $0.365 \pm 0.041$ \\
(f) p-eNOS & ox-LDL & $0.591 \pm 0.155$ \\
& ox-LDL + Ator & $0.809 \pm 0.032$ \\
& ox-LDL + Ator + Lv-shALOX15 & $0.994 \pm 0.084$ \\
(g) VCAM-1 & ox-LDL & $1.635 \pm 0.239$ \\
& ox-LDL + Ator + Lv-ALOX15 & $2.206 \pm 0.633$ \\
\hline \multirow{3}{*}{ (g) ICAM-1 } & ox-LDL & $1.435 \pm 0.152$ \\
& ox-LDL + Ator & $0.740 \pm 0.111$ \\
& ox-LDL + Ator + Lv-ALOX15 & $1.553 \pm 0.089$ \\
\hline
\end{tabular}

(Figure S2 in the online-only Supplementary Material). The cells treated with Lv-shALOX15 showed significantly reduced ALOX15 protein and 13-HODE expression in comparison with that of the Lv-GFP group (Figures 3(b) and 3(c), Table 3, $P<0.05)$, while the cells treated with Lv-ALOX15 showed significantly upregulated ALOX15 protein and 13-HODE expression in comparison with that of the Lv-GFP group (Figures 3(d) and 3(e) and Table 3, $P<0.01)$. However, ALOX15 mRNA was altered by Lv-shALOX15 but not Lv-ALOX15 (Figures S3(a)-3(d) in the online-only Supplementary Material). These results suggest that both ALOX15 protein expression and enzyme activity were altered after transfection with LvshALOX15 or Lv-ALOX15. As shown in Figures 3(f) and 3(g) and Table 3, changes in total Akt and total eNOS protein levels were not observed in the Lv-shALOX15 and Lv-ALOX15 groups. ox-LDL stimulation reduced protein levels of $\mathrm{p}$ $\mathrm{Akt}^{\text {Ser473 }}$ and $\mathrm{p}$-eNOS ${ }^{\text {Ser1177 }}(P<0.001)$ but upregulated protein expression levels of VCAM-1 and ICAM-1 $(P<$ 0.05 ), in comparison with the protein levels of the NC group. Atorvastatin treatment increased protein expression of $\mathrm{p}$ Akt $^{\text {Ser473 }}$ and p-eNOS ${ }^{\text {Ser1177 }}(P<0.05)$ but slightly decreased protein expression of VCAM-1 (no significant difference) and significantly decreased protein expression of ICAM$1(P<0.001)$, in comparison with the protein levels of the ox-LDL group. Silencing of ALOX15 slightly increased protein expression of $\mathrm{p}$-Akt ${ }^{\text {Ser473 }}$ (no significant difference) and increased protein expression of $\mathrm{p}-\mathrm{eNOS}^{\mathrm{Ser} 1177}(P<0.05)$ but slightly decreased protein expression levels of VCAM1 and ICAM-1 (no significant difference), in comparison with the protein levels of the Lv-GFP group. Interestingly, Lv-ALOX15 significantly decreased protein expression of p-Akt $^{\text {Ser473 }}(P<0.001)$ and slightly decreased protein expression of $\mathrm{p}$-eNOS ${ }^{\mathrm{Ser} 1177}$ (no significant difference) but significantly increased protein expression levels of VCAM1 and ICAM-1 $(P<0.01)$, in comparison with the protein levels of the Lv-GFP group. These results indicate that atorvastatin significantly increased protein levels of $\mathrm{p}-\mathrm{Akt} \mathrm{Ser}^{\mathrm{S} 43}$
TABLE 4: Numbers in results.

\begin{tabular}{lcc}
\hline Figure 4 & Group & Mean \pm SD \\
\hline \multirow{2}{*}{ (a) VCAM-1 } & ox-LDL & $1.249 \pm 0.111$ \\
& ox-LDL + PD146176 & $0.498 \pm 0.056$ \\
\hline \multirow{2}{*}{ (a) ICAM-1 } & ox-LDL & $2.012 \pm 0.362$ \\
& ox-LDL + PD146176 & $1.267 \pm 0.362$ \\
\hline \multirow{2}{*}{ (b) VCAM-1 } & ox-LDL + Lv-GFP & $1.118 \pm 0.078$ \\
& ox-LDL + Lv-shALOX15 & $0.916 \pm 0.016$ \\
\hline (b) ICAM-1 & ox-LDL + Lv-GFP & $1.437 \pm 0.147$ \\
& ox-LDL + Lv-shALOX15 & $0.632 \pm 0.056$ \\
\hline \multirow{2}{*}{ (c) p-AKT } & ox-LDL & $0.465 \pm 0.201$ \\
& ox-LDL + PD146176 & $0.814 \pm 0.134$ \\
\hline \multirow{2}{*}{ (c) p-eNOS } & ox-LDL & $0.428 \pm 0.145$ \\
& ox-LDL + PD146176 & $0.711 \pm 0.203$ \\
\hline (d) p-AKT & ox-LDL + Lv-GFP & $0.550 \pm 0.058$ \\
\hline \multirow{2}{*}{ (d) p-eNOS } & ox-LDL + Lv-shALOX15 & $1.113 \pm 0.289$ \\
& ox-LDL + Lv-GFP & $0.422 \pm 0.174$ \\
\hline
\end{tabular}

and p-eNOS ${ }^{\text {Ser1177 }}$ but decreased protein levels of adhesion molecules in HUVECs. Silencing of ALOX15 enhanced the effects of atorvastatin on protein levels of Akt, eNOS, and CAMs in vitro, whereas overexpression of ALOX15 attenuated these effects.

3.4. 15-LOX-1 Is Required for Upregulation of CAMs Expression and Inhibition of PI3K-Akt-eNOS Signaling under oxLDL Stimulation In Vitro. We next assessed the relationship between ALOX15, CAMs expression, and the PI3KAkt-eNOS signaling cascade under ox-LDL stimulation. Expression levels of VCAM-1 and ICAM-1 (Figure 4(a) and Table 4, $P<0.001$; Figure 4(b) and Table 4, $P<0.01$ ) were significantly increased in ox-LDL-stimulated HUVECs, but those of p-Akt ${ }^{\text {Ser473 }}$ and p-eNOS ${ }^{\text {Ser1177 }}$ (Figure $4(\mathrm{c})$ and Table 4, $P<0.005$; Figure 4(d) and Table 4, $P<0.05$ ) significantly decreased. In HUVECs, 15-LOX-1 inhibitor PD146176 abolished ox-LDL induced upregulation of VCAM1 and ICAM-1 (Figure 4(a) and Table 4, $P<0.001$ ), as well as downregulation of $\mathrm{p}$-Akt ${ }^{\mathrm{Ser} 473}$ and $\mathrm{p}$-eNOS ${ }^{\mathrm{Ser} 1177}$ (Figure 4(c) and Table 4, $P<0.05)$. Lv-shALOX15 blocked expression of VCAM-1/ICAM-1 (Figure 4(b) and Table $4, P<$ 0.001 ) under ox-LDL stimulation but activated expression of p-Akt ${ }^{\text {Ser473 }}$ and p-eNOS ${ }^{\text {Ser1177 }}$ (Figure 4(d) and Table 4, $P<0.05$ ), in comparison with the levels of the Lv-GFP group. These results suggest that ALOX15 is essential for ox-LDL induced enhancement of CAMs expression and inactivation of the PI3K-Akt-eNOS signaling cascade in vitro.

3.5. Atorvastatin Reduced 15-LOX-1 Expression via NF- $\kappa B$. In endothelial cells, ox-LDL activates diverse secondary messengers, including NF- $\kappa \mathrm{B}$ and AP-1, two oxidative stressresponsive transcription factors important in the regulation of cytokines, chemokines, and adhesion molecules [28]. As shown in Figure 5(a) and Table 5, the apparent degradation 

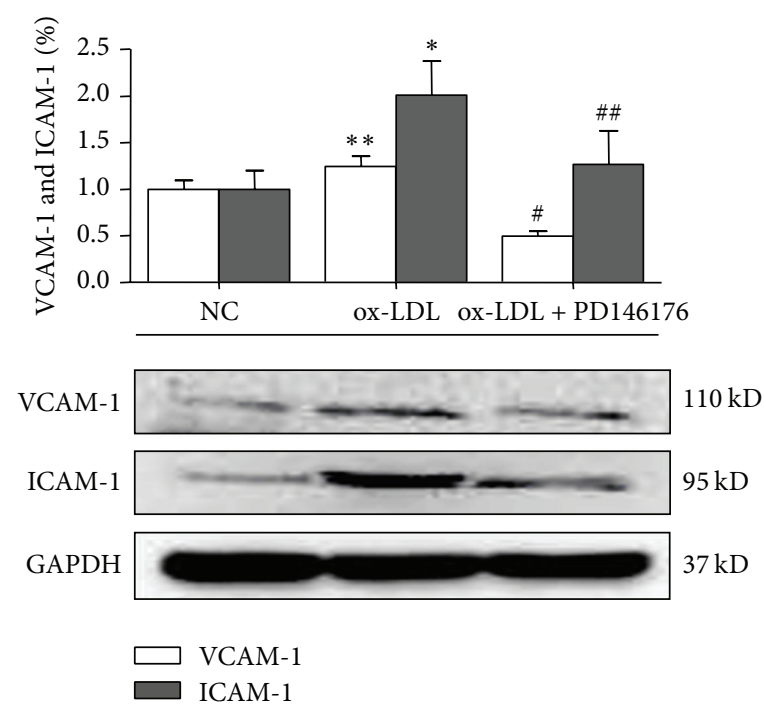

(a)
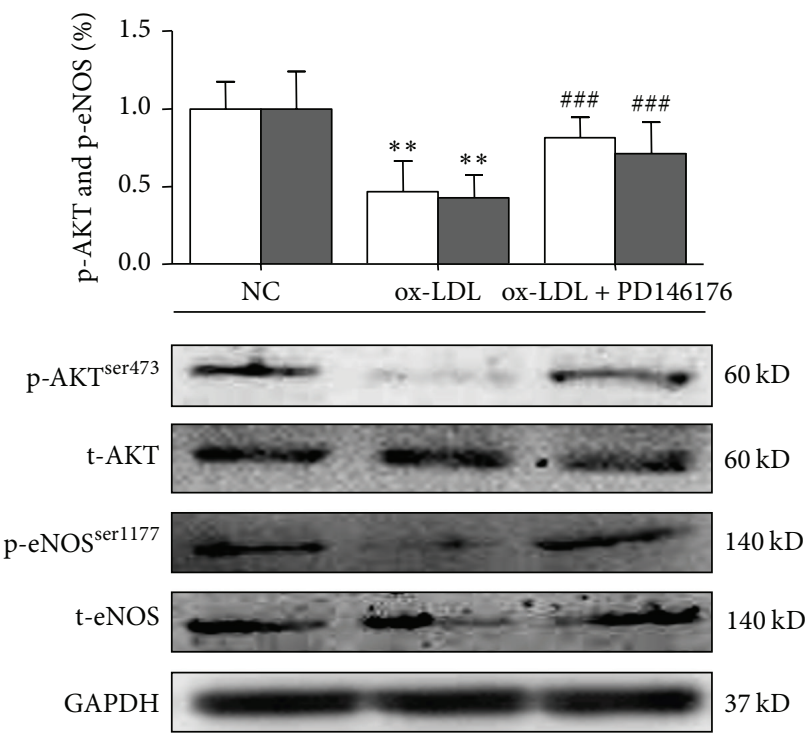

$\square$ p-AKT

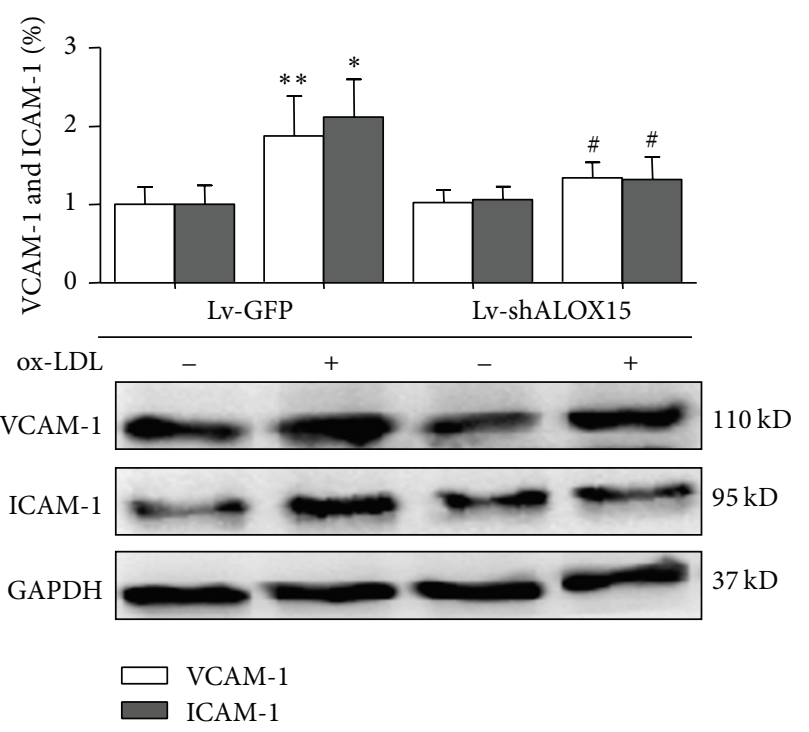

(b)

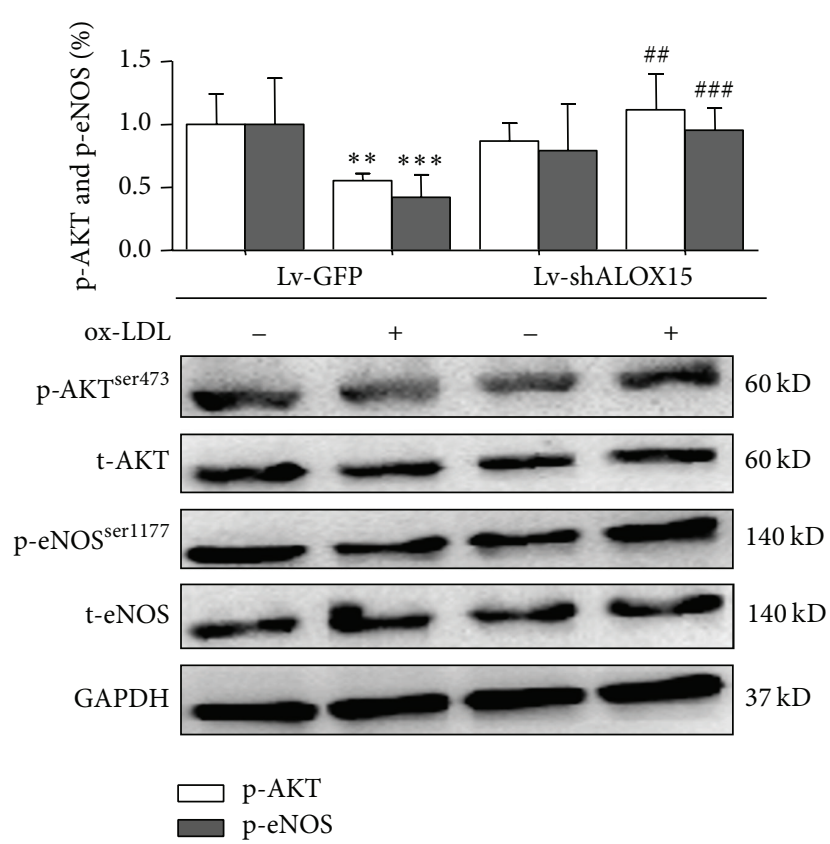

(d)

Figure 4: Effects of PD146176 $(20 \mu \mathrm{M})$ and Lv-shALOX15 on expression levels of VCAM-1, ICAM-1, AKT, and eNOS in HUVECs. (a) and (b) Western blot analysis of VCAM-1/ICAM-1 in HUVECs. (c) and (d) Western blot analysis of AKT/eNOS in HUVECs. Results are expressed as mean \pm SD. NC: normal control group. ${ }^{*} P<0.001,{ }^{* *} P<0.01$, and ${ }^{* * *} P<0.05$ versus the NC group; ${ }^{\#} P<0.001$, ${ }^{\# \#} P<0.01$, and ${ }^{\# \# \#} P<0.01$ versus the ox-LDL group or ox-LDL + Lv-GFP group. $N=5$ per group.

of $\mathrm{I} \kappa \mathrm{B}-\alpha$ in HUVECs in a time-dependent manner under oxLDL stimulation suggested enhanced NF- $\kappa$ B activity $(24 \mathrm{~h}$, $P<0.01 ; 48 \mathrm{~h}, P<0.05)$. As both 5-lipoxygenase and 12-lipoxygenase were identified to be transcriptionally regulated by NF- $\kappa \mathrm{B}$ [29], we measured ALOX15 expression in HUVECs under ox-LDL stimulation. To assess the role of $\mathrm{NF}-\kappa \mathrm{B}$ in regulation of LOX expression under ox-LDL stimulation, HUVECs were exposed to NF- $\kappa$ B inhibitor pyrrolidine dithiocarbamate (PDTC) at several concentrations. After $24 \mathrm{~h}$ of ox-LDL stimulation, ALOX15 protein and 13-HODE expression were significantly increased in comparison with those of the control group, but this effect was largely abolished by $10 \mu \mathrm{M}$ PDTC $(P<0.05)$ and $100 \mu \mathrm{M}$ PDTC $(P<0.001)$ (Figures 5(b) and 5(c) and Table 5). Consistently, qRT-PCR showed that PDTC treatment decreased ALOX15 mRNA expression (Figures S4(a) and 4(b) in the online-only Supplementary Material), suggesting that NF- $\kappa \mathrm{B}$ is an important mediator of ox-LDL-stimulated ALOX15 upregulation. As shown in Figure 5(d) and Table 5, atorvastatin treatment significantly reduced expression of NF- $\kappa$ B-p65 $(P<0.05)$ in 


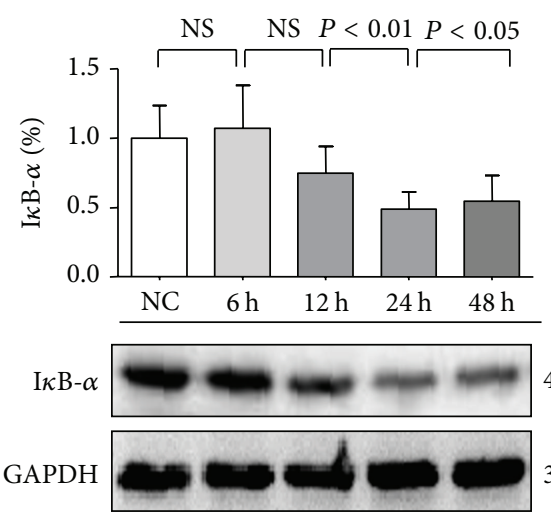

(a)

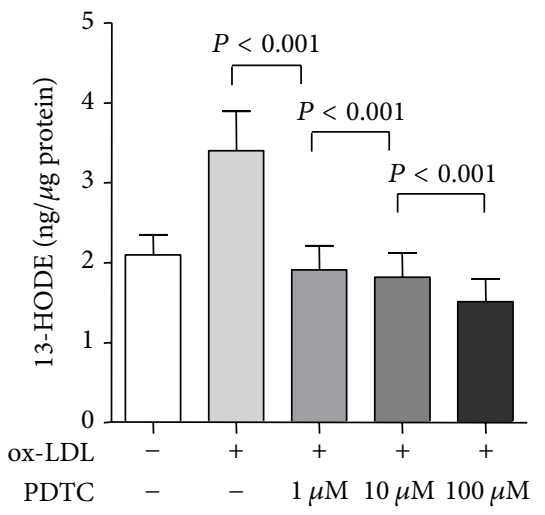

(c)

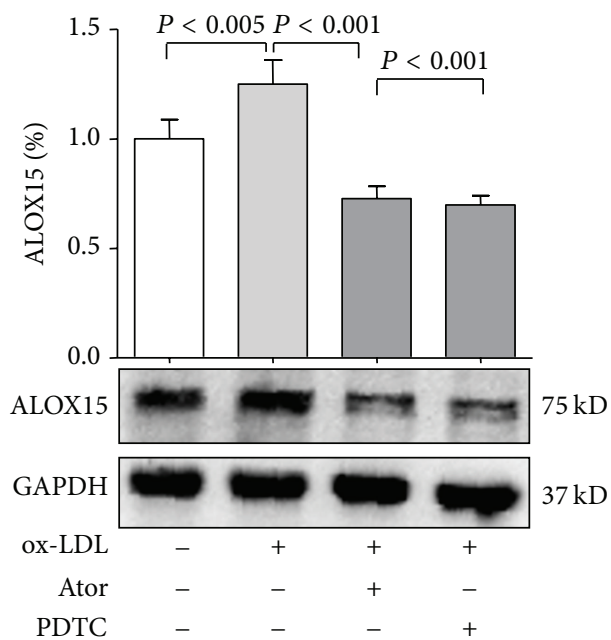

(e)

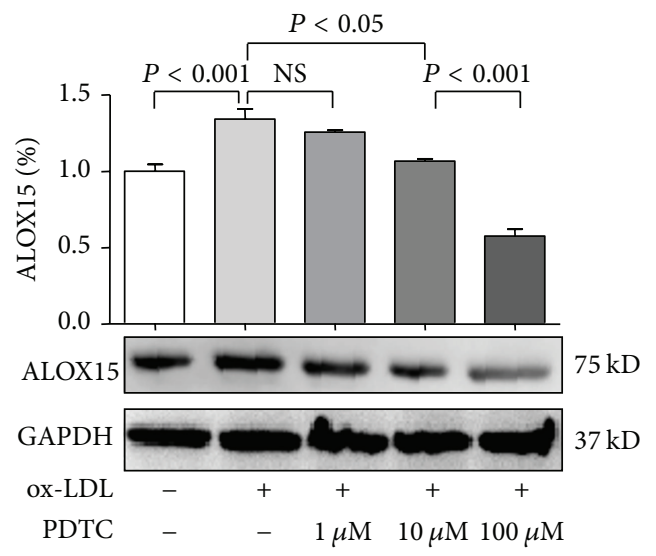

(b)

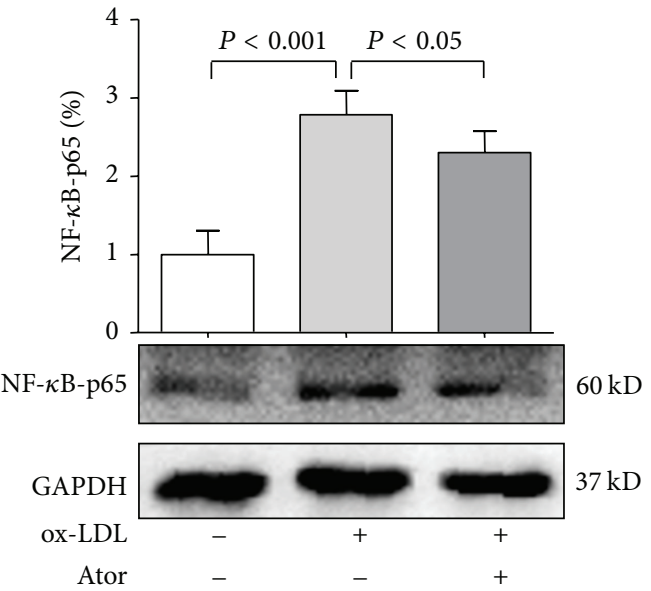

(d)

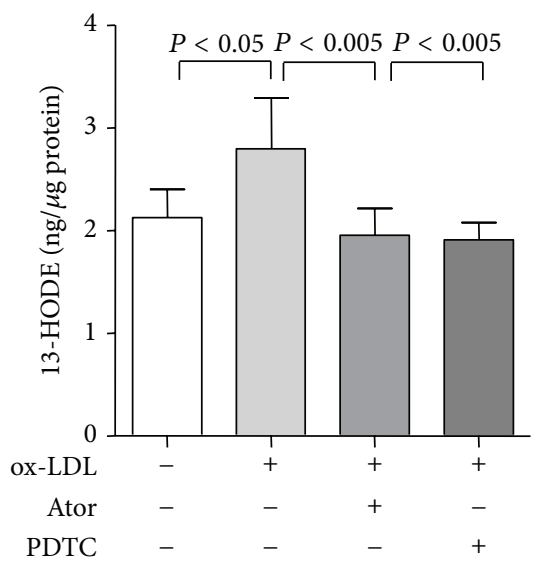

(f)

Figure 5: The relationship between atorvastatin and ALOX15. (a) ox-LDL (100 mg/L) increased NF- $\kappa$ B activity in a time-dependent manner. (b) and (c) NF- $\kappa$ B activity affected ox-LDL-induced expression of ALOX15 in HUVECs, which were preincubated with pyrrolidine dithiocarbamate (PDTC) $(1 \mu \mathrm{M}$ to $100 \mu \mathrm{M})$ for $1 \mathrm{~h}$ before ox-LDL stimulation. (d) HUVECs were preincubated with atorvastatin (10 $\mu \mathrm{M})$ for $8 \mathrm{~h}$ before ox-LDL stimulation, after which NF- $\kappa$ B-p65 protein levels were examined by western blot analysis. (e) and (f) Effects of atorvastatin and PDTC on ALOX15 protein expression and enzyme activity in HUVECs. Results are expressed as mean \pm SD. NC: normal control; NS: not significant. $N=3$ or 4 per group. 
TABLE 5: Numbers in results.

\begin{tabular}{lcc}
\hline Figure 5 & Group & Mean \pm SD \\
\hline (a) & $24 \mathrm{~h}$ & $0.491 \pm 0.123$ \\
& $48 \mathrm{~h}$ & $0.545 \pm 0.187$ \\
\hline (b) & ox-LDL + PD146176 $(10 \mu \mathrm{M})$ & $1.068 \pm 0.013$ \\
& ox-LDL + PD146176 $(100 \mu \mathrm{M})$ & $0.576 \pm 0.045$ \\
\hline & ox-LDL & $3.401 \pm 0.489$ \\
(c) & ox-LDL + PD146176 $(1 \mu \mathrm{M})$ & $1.192 \pm 0.297$ \\
& ox-LDL + PD146176 $(100 \mu \mathrm{M})$ & $1.819 \pm 0.298$ \\
& ox-LDL + PD146176 $(100 \mu \mathrm{M})$ & $1.510 \pm 0.285$ \\
\hline (d) & ox-LDL + Ator & $2.303 \pm 0.274$ \\
\hline (e) & ox-LDL + Ator & $0.727 \pm 0.056$ \\
& ox-LDL + PD146176 & $0.698 \pm 0.042$ \\
\hline & ox-LDL & $2.803 \pm 0.492$ \\
(f) & ox-LDL + Ator & $1.955 \pm 0.264$ \\
& ox-LDL + PD146176 & $1.911 \pm 0.172$ \\
\hline
\end{tabular}

ox-LDL-stimulated HUVECs. In addition, ox-LDL-induced ALOX15 protein and 13-HODE expression were inhibited by treatment with atorvastatin $(P<0.005)$ or PDTC $(P<$ 0.005 ) (Figures 5(e) and 5(f) and Table 5). In addition, qRTPCR showed that PDTC or atorvastatin treatment decreased ALOX15 mRNA expression (Figures S4(c) and 4(d) in the online-only Supplementary Material). These results suggest that inhibition of NF- $\kappa \mathrm{B}$ activation plays a role in the effects of atorvastatin on ALOX15 expression.

\section{Discussion}

In the present study, overexpression of ALOX15 in ballooninjured rat carotid arteries increased IMT. Atorvastatin treatment suppressed upregulation of adhesion molecules and downregulation of eNOS induced by balloon injury, whereas overexpression of ALOX15 partially eliminated these effects. Atorvastatin suppressed upregulation of adhesion molecules and downregulation of $\mathrm{p}$-Akt $\mathrm{ser}^{\text {se73 }}$ and $\mathrm{p}$-eNOS ${ }^{\text {ser1177 }}$ induced by ox-LDL stimulation in vitro. Silencing of ALOX15 enhanced these effects of atorvastatin on adhesion molecules, Akt, and eNOS, whereas overexpression of ALOX15 eliminated these effects. ALOX15 was required for upregulation of CAMs expression and downregulation of $\mathrm{p}-\mathrm{Akt}^{\mathrm{ser} 473}$ and p-eNOS ${ }^{\text {ser1177 }}$ expression under ox-LDL stimulation. NF$\kappa \mathrm{B}$ was essential for expression of ALOX15 induced by oxLDL. Atorvastatin might reduce expression of ALOX15 via NF- $\kappa \mathrm{B}$. To the best of our knowledge, these results provide the evidence that statin treatment might modulate CAMs expression and the PI3K-Akt-eNOS signaling cascade by inactivating ALOX15. These results suggest that ALOX15 is involved in the effect of atorvastatin on endothelial function.

4.1. Relationship between ALOX15 and the Effect of Atorvastatin on Endothelial Function. On account of minor human ALOX15 mRNA that were detected in atherosclerotic lesions, ALOX15 may play a role in the initiation of atherosclerosis but not in later stages of atherogenesis [9]. Our study demonstrates that ALOX15 overexpression markedly increases IMT, suggesting that ALOX15 plays a critical role in endothelial proliferation after arterial injury. After observing the detrimental effects of ALOX15 on the injured vessel segment, we investigated the relationship between ALOX15 and atorvastatin. In the atorvastatin-treated rats, protein levels of eNOS, p-Akt ${ }^{\text {ser473 }}$, and p-eNOS ${ }^{\text {ser1177 }}$ were increased, but protein levels of VCAM-1 and ICAM-1 were decreased. Overexpression of ALOX15 inhibited the beneficial effects of atorvastatin on eNOS, p-Akt ${ }^{\text {ser473 }}$, p-eNOS ${ }^{\text {ser1177 }}$, VCAM-1, and ICAM-1. The observed upregulation of eNOS and downregulation of VCAM-1 and ICAM-1 suggest that atorvastatin may have favorable effects on endothelial function and inflammation, confirming previous studies [30]. Consistent results were observed in vitro, where silencing of ALOX15 enhanced the effects of atorvastatin on endothelial function. We demonstrate here that the effects of atorvastatin on vascular endothelial function and inflammation are associated with regulation of ALOX15 expression.

4.2. Effect of ALOX15/ox-LDL on Adhesion Molecules and PI3K-Akt-eNOS Signaling. In human endothelial cells, oxLDL increased expression of VCAM-1 and ICAM-1 but reduced expression of $\mathrm{p}-\mathrm{Akt}^{\mathrm{ser} 473}$ and $\mathrm{p}$-eNOS ${ }^{\mathrm{ser} 1177}$. The stimulatory effects of ox-LDL were observed at the protein level in HUVECs; interestingly, these effects were suppressed by ALOX15 inhibitors or ALOX15-shRNA. Emerging evidence suggests that ox-LDL exposure is associated with upregulation of ICAM-1 and VCAM-1, as well as inactivation of the PI3K-Akt-eNOS signaling cascade in multiple cell types; however, the mechanism by which adhesion molecules and Akt/eNOS are regulated in ox-LDL-induced endothelial dysfunction has not been fully elucidated [31-33]. The present study indicates that ALOX15 is involved in regulation of CAMs expression and the PI3K-Akt-eNOS signaling cascade in HUVECs under ox-LDL stimulation.

4.3. Role of $N F-\kappa B$ in ox-LDL-Induced Upregulation of ALOX15. Previous studies have demonstrated that ox-LDL mainly activates NF- $\kappa \mathrm{B}$, an oxidative stress-responsive transcription factor important in the regulation of cytokines, chemokines, and inflammatory molecules in endothelial cells, including 5-lipoxygenase and 12-lipoxygenase [29, 34, 35]. Although our work, similar to previous works, showed reduced $\mathrm{I} \kappa \mathrm{B}-\alpha$ activity in HUVECs under ox-LDL stimulation, we also demonstrated that ox-LDL induced upregulation of ALOX15 and NF- $\kappa$ B, which was necessary for ox-LDL-induced ALOX15 expression. Moreover, atorvastatin inhibited overexpression of NF- $\kappa \mathrm{B}$ and ALOX15 induced by ox-LDL stimulation in HUVECs, while inhibitors of atorvastatin and NF- $\kappa \mathrm{B}$ had similar effects on ALOX15 expression. A large number of proinflammatory genes were modulated by $\mathrm{NF}-\kappa \mathrm{B}$, which can be activated by ox-LDL. Therefore, NF- $\kappa \mathrm{B}$ activation might be involved in ox-LDL-induced upregulation of VCAM-1 and ICAM- 1 and downregulation of Akt and eNOS. The role of statins as inhibitors of NF- $\kappa$ B has also been investigated in cellular and animal models. Exposure of vascular smooth muscle cells (VSMCs) to atorvastatin prevents TNF- $\alpha$ and angiotensin II-induced activation 
of $\mathrm{NF}-\kappa \mathrm{B}$ and subsequent upregulation of inflammatory mediators [36]. Simvastatin and atorvastatin attenuate oxLDL-induced activation of NF- $\kappa \mathrm{B}$ in human coronary artery endothelium cells [37]. In the present paper, ox-LDL led to regulation of CAMs and eNOS; then these effects were inhibited by blocking ALOX15. Actually, we observed similar phenomenon by blocking ALOX15; hence we considered ox-LDL as a stimulation factor to study the relationship between ALOX15 and atorvastatin step by step. Simvastatin treatment reduces $\mathrm{NF}-\kappa \mathrm{B}$ activity in atherosclerotic plaques and circulating mononuclear cells in animals with dietinduced atherosclerosis independently of its lipid-lowering effects [38]. In agreement with previous studies, the present study indicates that atorvastatin treatment reduces $\mathrm{NF}-\kappa \mathrm{B}$ expression. In addition, inhibition of $N F-\kappa B$ induced by treatment with atorvastatin might decrease the activity of ALOX15.

\section{Additional Points}

Limitations of the Present Study. The present study demonstrates that atorvastatin reduces inflammation and restores function in endothelial cells via a process involving modulation of CAMs expression and the PI3K/Akt/eNOS signaling cascade by NF- $\kappa \mathrm{B}$ and ALOX15. However, according to previous expression studies, levels of ALOX15 mRNA are only present in small amounts in advanced atherosclerotic lesions. Thus, despite the discovery of the interaction between ALOX15 and atorvastatin, it is unclear whether or not atorvastatin effects in the present study can indeed explain the beneficial cardiovascular effects of statins in humans. Further study is still needed to clarify this novel pathway. In summary, the results of this study provide insight into the initial pathogenesis of AS and guidance for the development of new therapeutic strategies for the treatment of AS.

\section{Competing Interests}

All authors declare that there is no conflict of interests.

\section{Acknowledgments}

This work was supported by the National Natural Science Foundation (Grant nos. 81100196 and 81570212), the Natural Science Foundation Project of CQ CSTC (Grant no. CSTC, 2011BB5133), and a Pfizer Pharmaceutical Ltd. Competition Grant (ws1790576). The authors greatly appreciate Chunli Li and Dezhang Zhao (Institute of Life Sciences, Chongqing Medical University) for their excellent technical support regarding the flow cytometry and western blot analyses.

\section{References}

[1] A. M. Lefer, B. Campbell, Y.-K. Shin, R. Scalia, R. Hayward, and D. J. Lefer, "Simvastatin preserves the ischemic-reperfused myocardium in normocholesterolemic rat hearts," Circulation, vol. 100, no. 2, pp. 178-184, 1999.

[2] D. Pruefer, R. Scalia, and A. M. Lefer, "Simvastatin inhibits leukocyte-endothelial cell interactions and protects against inflammatory processes in normocholesterolemic rats," Arteriosclerosis, Thrombosis, and Vascular Biology, vol. 19, no. 12, pp. 2894-2900, 1999.

[3] J. Dupuis, J.-C. Tardif, P. Cernacek, and P. Théroux, "Cholesterol reduction rapidly improves endothelial function after acute coronary syndromes: the RECIFE (Reduction of Cholesterol in Ischemia and Function of the Endothelium) trial," Circulation, vol. 99, no. 25, pp. 3227-3233, 1999.

[4] I. Kosmidou, J. P. Moore, M. Weber, and C. D. Searles, "Statin treatment and $3^{\prime}$ polyadenylation of eNOS mRNA," Arteriosclerosis, Thrombosis, and Vascular Biology, vol. 27, no. 12, pp. 26422649, 2007.

[5] D. Sumi, T. Hayashi, N. K. Thakur et al., "A HMG-CoA reductase inhibitor possesses a potent anti-atherosclerotic effect other than serum lipid lowering effects-the relevance of endothelial nitric oxide synthase and superoxide anion scavenging action," Atherosclerosis, vol. 155, no. 2, pp. 347-357, 2001.

[6] Z. Taoufiq, P. Pino, N. N'dilimabaka et al., "Atorvastatin prevents Plasmodium falciparum cytoadherence and endothelial damage," Malaria Journal, vol. 10, article 52, 2011.

[7] M. Romano, L. Diomede, M. Sironi et al., "Inhibition of monocyte chemotactic protein-1 synthesis by statins," Laboratory Investigation, vol. 80, no. 7, pp. 1095-1100, 2000.

[8] S. Yamamoto, "Mammalian lipoxygenases: molecular structures and functions," Biochimica et Biophysica Acta (BBA)-Lipids and Lipid Metabolism, vol. 1128, no. 2-3, pp. 117-131, 1992.

[9] H. Kuhn, S. Banthiya, and K. Van Leyen, "Mammalian lipoxygenases and their biological relevance," Biochimica et Biophysica Acta (BBA)-Molecular and Cell Biology of Lipids, vol. 1851, no. 4, pp. 308-330, 2015.

[10] C. D. Funk, X.-S. Chen, E. N. Johnson, and L. Zhao, "Lipoxygenase genes and their targeted disruption," Prostaglandins and Other Lipid Mediators, vol. 68-69, pp. 303-312, 2002.

[11] J. Wittwer and M. Hersberger, "The two faces of the 15lipoxygenase in atherosclerosis," Prostaglandins Leukotrienes and Essential Fatty Acids, vol. 77, no. 2, pp. 67-77, 2007.

[12] S. J. A. Wuest, M. Crucet, C. Gemperle, C. Loretz, and M. Hersberger, "Expression and regulation of 12/15-lipoxygenases in human primary macrophages," Atherosclerosis, vol. 225, no. 1, pp. 121-127, 2012.

[13] R. Natarajan and J. L. Nadler, "Lipid inflammatory mediators in diabetic vascular disease," Arteriosclerosis, Thrombosis, and Vascular Biology, vol. 24, no. 9, pp. 1542-1548, 2004.

[14] C. Sultana, Y. Shen, V. Rattan, and V. K. Kalra, "Lipoxygenase metabolites induced expression of adhesion molecules and transendothelial migration of monocyte-like HL-60 cells is linked to protein kinase C activation," Journal of Cellular Physiology, vol. 167, no. 3, pp. 477-487, 1996.

[15] R. Natarajan, M. A. Reddy, K. U. Malik, S. Fatima, and B. V. Khan, "Signaling mechanisms of nuclear factor- $\kappa \mathrm{B}$-mediated activation of inflammatory genes by 13 -hydroperoxyoctadecadienoic acid in cultured vascular smooth muscle cells," Arteriosclerosis, Thrombosis, and Vascular Biology, vol. 21, no. 9, pp. 1408-1413, 2001.

[16] A. D. Dobrian, D. C. Lieb, B. K. Cole, D. A. Taylor-Fishwick, S. K. Chakrabarti, and J. L. Nadler, "Functional and pathological roles of the 12- and 15-lipoxygenases," Progress in Lipid Research, vol. 50, no. 1, pp. 115-131, 2011.

[17] S. M. Green-Mitchell, S. A. Tersey, B. K. Cole et al., "Deletion of 12/15-lipoxygenase alters macrophage and islet function in NOD-Alox15null mice, leading to protection against type 1 
diabetes development," PLoS ONE, vol. 8, no. 2, Article ID e56763, 2013.

[18] E. D. Sezer, E. Y. Sozmen, D. Nart, and T. Onat, "Effect of atorvastatin therapy on oxidant-antioxidant status and atherosclerotic plaque formation," Vascular Health and Risk Management, vol. 7, no. 1, pp. 333-343, 2011.

[19] D. A. Tulis, "Rat carotid artery balloon injury model," Methods in Molecular Medicine, vol. 139, pp. 1-30, 2007.

[20] C. M. Dollery, S. E. Humphries, A. McClelland, D. S. Latchman, and J. R. McEwan, "In vivo adenoviral gene transfer of TIMP-1 after vascular injury reduces neointimal formation," Annals of the New York Academy of Sciences, vol. 878, pp. 742-743, 1999.

[21] J. M. Yang, Y. Wang, L. H. Qi et al., "Combinatorial interference of toll-like receptor 2 and 4 synergistically stabilizes atherosclerotic plaque in apolipoprotein E-knockout mice," Journal of Cellular and Molecular Medicine, vol. 15, no. 3, pp. 602-611, 2011.

[22] M. W. Liu, G. S. Roubin, and S. B. King III, "Restenosis after coronary angioplasty: potential biologic determinants and role of intimal hyperplasia," Circulation, vol. 79, no. 6, pp. 1374-1387, 1989.

[23] K. Kusaba, H. Kai, M. Koga et al., "Inhibition of intrinsic interferon- $\gamma$ function prevents neointima formation after balloon injury," Hypertension, vol. 49, no. 4, pp. 909-915, 2007.

[24] S. N. Murthy, C. V. Desouza, N. W. Bost et al., "Effects of salsalate therapy on recovery from vascular injury in female zucker fatty rats," Diabetes, vol. 59, no. 12, pp. 3240-3246, 2010.

[25] P. Giuseppe, C. Massimo, P. Vincenzo et al., "Protection from procedural myocardial injury by atorvastatin is associated with lower levels of adhesion molecules after percutaneous coronary intervention," Journal of the American College of Cardiology, vol. 48, pp. 1560-1566, 2006.

[26] S. Parthasarathy, A. Raghavamenon, M. O. Garelnabi, and N. Santanam, "Oxidized low-density lipoprotein," Methods in Molecular Biology, vol. 610, pp. 403-417, 2010.

[27] S. Parthasarathy, N. Santanam, S. Ramachandran, and O. Meilhac, "Oxidants and antioxidants in atherogenesis. An appraisal," Journal of Lipid Research, vol. 40, no. 12, pp. 2143-2157, 1999.

[28] A. J. Valente, A. M. Irimpen, U. Siebenlist, and B. Chandrasekar, "OxLDL induces endothelial dysfunction and death via TRAF3IP2: inhibition by HDL3 and AMPK activators," Free Radical Biology and Medicine, vol. 70, pp. 117-128, 2014.

[29] H. L. Pahl, "Activators and target genes of Rel/NF- $\kappa$ B transcription factors," Oncogene, vol. 18, no. 49, pp. 6853-6866, 1999.

[30] M. Margaritis, K. M. Channon, and C. Antoniades, "Statins as regulators of redox state in the vascular endothelium: beyond lipid lowering," Antioxidants and Redox Signaling, vol. 20, no. 8, pp. 1198-1215, 2014.

[31] T. Shen, Y. Zhu, J. Patel et al., “T-Box20 suppresses oxidized low-density lipoprotein-induced human vascular endothelial cell injury by upregulation of PPAR- $\gamma$," Cellular Physiology and Biochemistry, vol. 32, no. 5, pp. 1137-1150, 2013.

[32] S. C. Tai, G. B. Robb, and P. A. Marsden, "Endothelial nitric oxide synthase: a new paradigm for gene regulation in the injured blood vessel," Arteriosclerosis, Thrombosis, and Vascular Biology, vol. 24, no. 3, pp. 405-412, 2004.

[33] F. Y. Lin, N. W. Tsao, C. M. Shih et al., "The biphasic effects of oxidized-low density lipoprotein on the vasculogenic function of endothelial progenitor cells," PLOS ONE, vol. 10, Article ID e0123971, 2015.

[34] T. Sawamura, N. Kume, T. Aoyama et al., "An endothelial receptor for oxidized low-density lipoprotein,” Nature, vol. 386, no. 6620 , pp. 73-77, 1997.
[35] J. L. Mehta and D. Y. Li, "Identification and autoregulation of receptor for OX-LDL in cultured human coronary artery endothelial cells," Biochemical and Biophysical Research Communications, vol. 248, no. 3, pp. 511-514, 1998.

[36] M. Ortego, C. Bustos, M. A. Hernández-Presa et al., "Atorvastatin reduces NF- $\kappa$ B activation and chemokine expression in vascular smooth muscle cells and mononuclear cells," Atherosclerosis, vol. 147, no. 2, pp. 253-261, 1999.

[37] D. Li, H. Chen, F. Romeo, T. Sawamura, T. Saldeen, and J. L. Mehta, "Statins modulate oxidized low-density lipoproteinmediated adhesion molecule expression in human coronary artery endothelial cells: role of LOX-1," Journal of Pharmacology and Experimental Therapeutics, vol. 302, no. 2, pp. 601-605, 2002.

[38] M. A. Hernández-Presa, M. Ó. Ortego, J. Tuñón et al., "Simvastatin reduces NF- $\kappa \mathrm{B}$ activity in peripheral mononuclear and in plaque cells of rabbit atheroma more markedly than lipid lowering diet," Cardiovascular Research, vol. 57, no. 1, pp. 168177,2003 


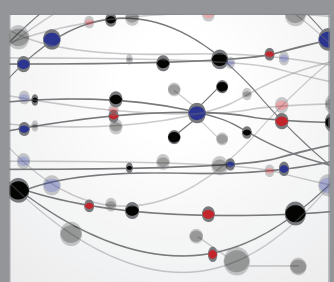

The Scientific World Journal
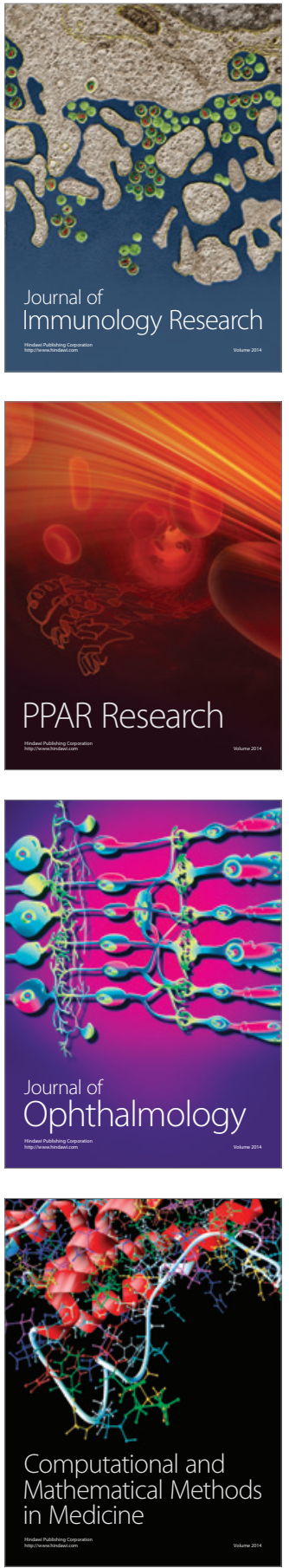

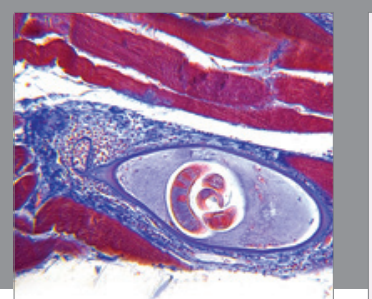

Gastroenterology Research and Practice

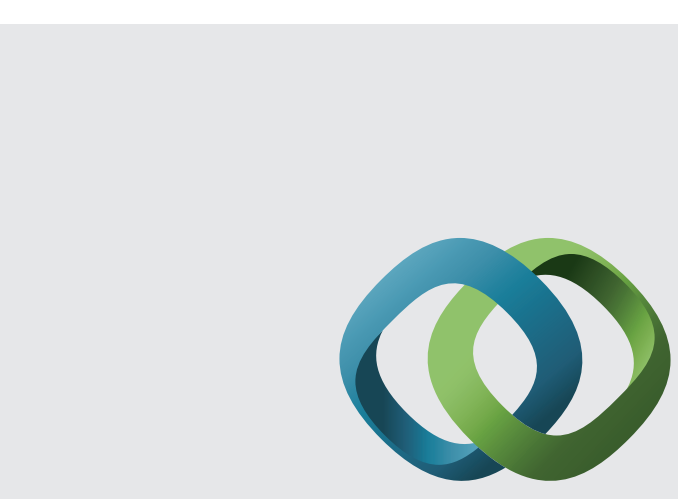

\section{Hindawi}

Submit your manuscripts at

http://www.hindawi.com
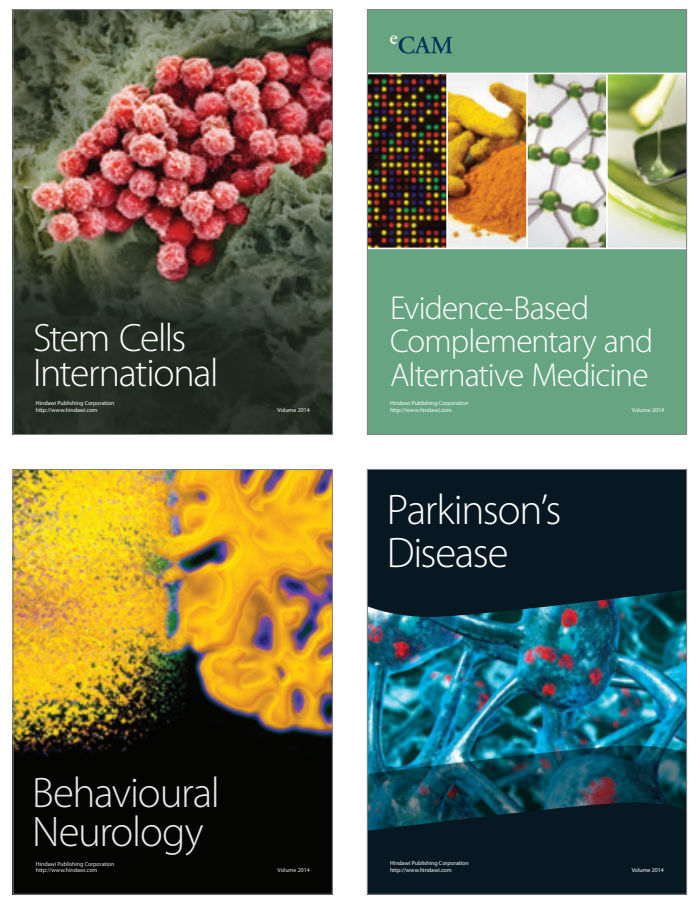
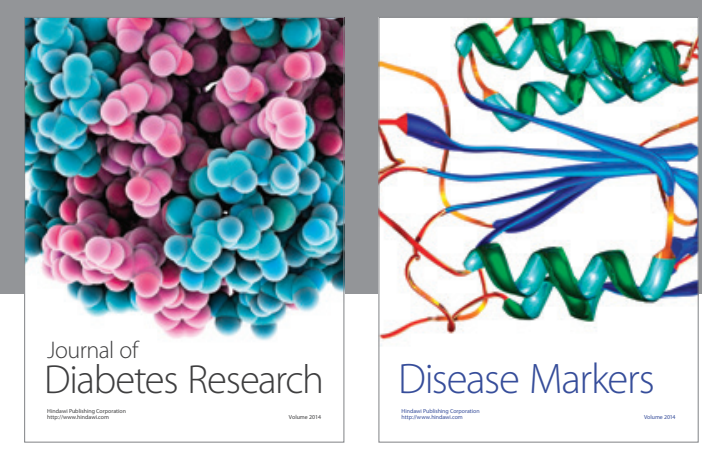

Disease Markers
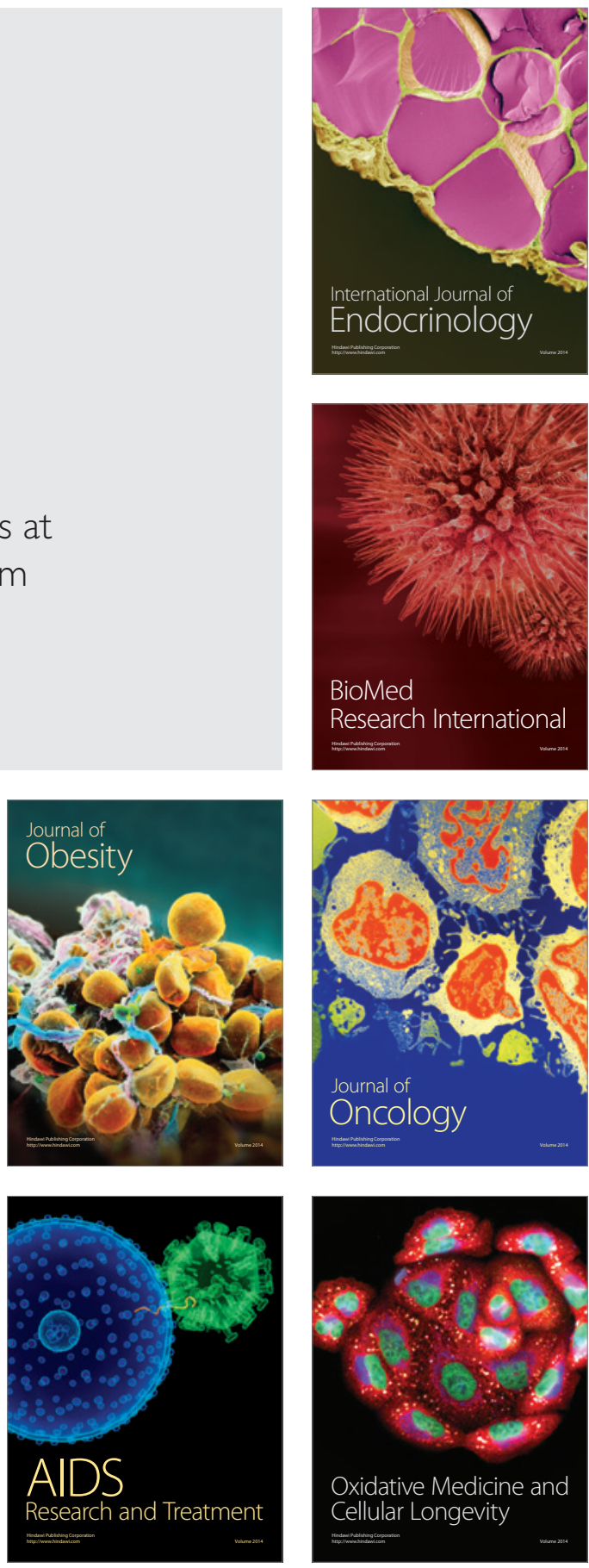